\title{
Die Klagbarkeit gesellschaftsrechtlicher Treuepflichten
}

\author{
Inhaltsübersicht
}

I. Einführung

II. Problemstellung

1. Die Klagbarkeit der Stimmpflicht der Gesellschafter

2. Die Klagbarkeit des Wettbewerbsverbots von Geschäftsleitern in der Kapitalgesellschaft \& Co. KG

a) Die Gruner + Jahr-Entscheidung des BGH

b) BGH: Drittschutz nicht auf Primärebene (Unterlassung), sondern nur auf Sekundärebene (Schadensersatz)

3. Befund: Keine stringente Klagbarkeitsdogmatik bei gesellschaftsrechtlichen Treuepflichten

III. These

1. Rekurs auf die allgemeine Klagbarkeitsdogmatik von Rücksichtspflichten

2. Begründung

IV. Normative Verankerung der Treuepflichtdogmatik in $\S \S 241,242$ BGB 1. Schuldrechtliche Treuepflicht

2. Mitgliedschaftliche Treuepflicht
3. Organschaftliche Treuepflicht

4. Zwischenbilanz

V. Die Klagbarkeit von Rücksichtspflichten (§ 241 Abs. 2 BGB)

1. Die Einzelelemente eines Schuldverhältnisses

2. Die Klagbarkeit als Eigenschaft von Leistungspflichten

3. Die Klagbarkeit als Eigenschaft von Rücksichtspflichten?

a) Entwicklung und Meinungsstand aa) Die Doktrin Lehmanns und Sibers

bb) Position der Rechtsprechung

cc) Literatur: Bejahung der Klagbarkeit unter besonderen Voraussetzungen

b) Voraussetzungen der Klagbarkeit aa) Konkretisierbarkeit des Pflichtinhalts

bb) Überwiegendes schutzwürdiges Interesse

VI. Lösung der Eingangsbeispiele

VII. Zusammenfassung in Thesen

\section{Einführung}

Die Treuepflichten sind von Rechtsprechung und Wissenschaft zu einem der wesentlichen Institute des Gesellschaftsrechts ausgebaut worden ${ }^{1}$. Sie haben sich zu einem "zentralen Rechtssatz"2 in Form einer "Generalklausel“3 entwickelt. Martin Winter hatte daran mit seiner wegweisenden Monographie zu

1 Vgl. aus jüngerer Zeit z. B. Merkt in MünchKomm. GmbHG, 2010, 13 Rz. 88 ff.; Verse, Treuepflichten und Gleichbehandlungsgrundsatz, in Bayer/Habersack (Hrsg.), Aktienrecht im Wandel, Bd. II, 2007, S. 579 ff.; Weller in Bork/Schäfer, GmbHG, 2010, $\S 13 \mathrm{Rz} .7 \mathrm{ff}$.

2 Ulmer in MünchKomm. BGB, 5. Aufl. 2009, § 705 Rz. 221.

3 Wiedemann, WM 2009, $1 \mathrm{ff}$. 
den „Mitgliedschaftlichen Treuebindungen im GmbH-Recht" (1988) ${ }^{4}$ einen entscheidenden Anteil. Martin Winter war dem Verfasser, der von 2002 - 2005 als Anwalt im Mannheimer Büro der internationalen Sozietät Shearman \& Sterling tätig gewesen ist, ein großes Vorbild, insbesondere darin, anwaltliche und wissenschaftliche Exzellenz zu vereinen. Auch seine freundschaftliche Art und seine emotionale Seite bleiben unvergessen. Die folgenden Zeilen seien ihm gewidmet.

Innerhalb der gesellschaftsrechtlichen Treuebindungen ist zwischen den organschaftlichen Treuepflichten der Geschäftsleiter und den mitgliedschaftlichen Treuepflichten der Gesellschafter zu unterscheiden ${ }^{5}$. Diese Unterscheidung betrifft die Rechtsnatur der Treuepflichten. Sie geht auf die allgemeine Vertragsrechtsdogmatik zurück. Im Anschluss an die grundlegenden Arbeiten von Rumpf $^{6}$, Beyerle ${ }^{7}$ und Würdinger ${ }^{8}$ werden Verträge heutzutage je nach der ihnen zugrunde liegenden Interessenstruktur in Verträge des Interessengegensatzes, der Interessengemeinschaft und der Fremdinteressenwahrung eingeordnet $^{9}$.

Diese Einordnung hat nicht nur dogmatischen Wert, sondern geht auch mit unterschiedlichen Rechtsfolgen einher. So ist die organschaftliche Treuepflicht der Geschäftsleiter aufgrund ihrer ausschließlichen Fremdinteressenfokussierung intensiver als die mitgliedschaftliche Treuepflicht der Gesellschafter ${ }^{10}$. Dies zeigt sich an gewissen Ausprägungen der Treuepflicht, etwa an der Vermögensbetreuungspflicht im strafrechtlichen Untreuetatbestand ( $\$ 266$ StGB), welche nur die Geschäftsleiter, nicht aber die Gesellschafter trifft ${ }^{11}$, sowie am Wettbewerbsverbot. Dieses ist für Geschäftsleiter deutlich strenger als für Gesellschafter ${ }^{12}$. Während ein Gesellschafter einer personalistisch strukturierten

4 Zur gewichtigen, weit über eine „normale“ Dissertation hinausreichenden Bedeutung dieser Schrift Zöllner, ZIP-Sonderbeilage zu Heft 39/2010, S. 3 f., auch hier abgedruckt S. 1 ff.

5 Fleischer, WM 2003, 1045, 1046 f.; Hüffer, AktG, 9. Aufl. 2010, § 84 Rz. 9; Wiedemann, WM 2009, 1 ff. Näher infra unter IV.2. und IV.3.

6 Rumpf, AcP 119 (1921), 1, 53 ff., systematisiert die Schuldverträge erstmals nach Parteiinteressen.

7 Beyerle, Die Treuhand im Grundriss des deutschen Privatrechts, 1932, S. 46, differenziert zwischen den drei Grundtatbeständen Synallagma, Gesamthand und Treuhand.

8 Würdinger, Gesellschaften, Bd. I, 1937, S. 9 ff., unterscheidet nach Verträgen des Interessengegensatzes (mea res agitur), der Interessengemeinschaft (nostra res agitur) und der Interessenwahrung (tua res agitur).

9 Martinek in Staudinger, BGB, 2006, Vorbem. zu $§ \S 662$ ff. Rz. 24 ff.; Rittner, Ausschließlichkeitsbindungen, 1957, S. 112 ff., 141 ff., $160 \mathrm{ff.}$.; Ulmer, Der Vertragshändler, 1969, S. 265 ff.; Ulmer in MünchKomm. BGB, 5. Aufl. 2009, Vor § 705 Rz. 104 ff.; Wiedemann, Das Arbeitsverhältnis als Austausch- und Gemeinschaftsverhältnis, 1966, S. 9 ff., 26 ff., 29; Weller, Die Vertragstreue, 2009, S. 197 ff.

10 Fleischer, WM 2003, 1045, 1047; Hellgardt, ZIP 2007, 2248, 2250; Weller, ZHR 175 (2011), 110, $127 \mathrm{f}$.

11 Schönke/Schröder/Perron, StGB, 28. Aufl. 2010, § 266, Rn. 25 f.; MünchKommStGB/ Dierlamm, 2006, §266, Rn. 83, 84.

12 Ausführlich Weller, ZHR 175 (2011), 110, $127 \mathrm{f}$. 
Gesellschaft im selben Geschäftszweig keine Konkurrenz machen darf (exemplarisch: $\S 112$ Abs. $1 \mathrm{HGB}$ ), reicht das Wettbewerbsverbot der Geschäftsleiter weiter. Letztere sind nicht nur verpflichtet, der Gesellschaft keine Konkurrenz im selben Geschäftszweig zu machen, sondern dürfen überhaupt nicht anderweitig tätig werden (beispielhaft: $\S 88$ Abs. 1 Satz 1 1. Alt. und Satz 2 AktG). Denn aufgrund des Fremdinteressenwahrungscharakters des Geschäftsleiteranstellungsvertrages sind sie gehalten, ihre Arbeitskraft der Gesellschaft ungeschmälert zukommen zu lassen ${ }^{13}$.

\section{Problemstellung}

Auch wenn die gesellschaftsrechtliche Treuepflichtdogmatik bereits eine beachtliche Durchdringung erfahren hat, so gibt es gleichwohl noch Forschungsbedarf, unter anderem im Hinblick auf die Rechtsfolgen von Treuepflichten auf Primär- und Sekundärebene ${ }^{14}$. Nicht hinreichend geklärt ist insbesondere die Frage, ob Treuepflichten auf Primärebene stets als solche in Natur durchgesetzt werden können, wie es etwa bei Leistungspflichten (§ 241 Abs. 1 BGB) grundsätzlich der Fall ist ${ }^{15}$, oder ob sie lediglich auf Sekundärebene Rechtsfolgen in Form von Schadensersatz im Verletzungsfall zeitigen. Die Unschärfe der Treuepflichtdogmatik auf Rechtsfolgenebene sei an zwei Beispielen erhellt:

\section{Die Klagbarkeit der Stimmpflicht der Gesellschafter}

Anerkannt ist, dass ein Gesellschafter aufgrund der mitgliedschaftlichen Treuepflicht gehalten sein kann, das Stimmrecht in einer bestimmten Art und Weise auszuüben ${ }^{16}$. Die Verpflichtung kann soweit gehen, einer konkreten Beschlussvorlage zuzustimmen ${ }^{17}$, etwa in dringenden Sanierungsfällen ${ }^{18}$. Zustimmungspflichten wurden ferner bejaht für das Ausscheiden eines in persönliche Zahlungsschwierigkeiten geratenen Gesellschafters ${ }^{19}$, zum Zweck der

13 Fleischer in Spindler/Stilz, AktG, 2. Aufl. 2010, § 88 Rz. 2; ders., WM 2003, 1045, $1050 \mathrm{f}$.

$14 \mathrm{Vgl}$ zu den verschiedenen Rechtsfolgen der Treuepflicht Merkt in MünchKomm. GmbHG, 2010, § $13 \mathrm{Rz} .186 \mathrm{ff}$.

15 Weller, Die Vertragstreue, 2009, S. 232 ff., 264, $381 \mathrm{ff}$.

16 Hueck/Fastrich in Baumbach/Hueck, GmbHG, 19. Aufl. 2010, § 13 Rz. 29.

17 BGH, Urt. v. 25.9.1986 - II ZR 262/85, BGHZ 98, 276, 278 ff.; BGH, Urt. v. 23.3.1987 II ZR 244/86, BB 1987, 1200; OLG Hamm, Urt. v. 9.12.1991 - 8 U 78/91, GmbHR 1992,612 .

18 BGH, Urt. v. 19.10.2009 - II ZR 240/08 ("Sanieren oder Ausscheiden“), BGHZ 183, 1 ff. = DStR 2009, 2495 f., s. dazu K. Schmidt, JZ 2010, 125; Weber, DStR 2010, 702.

19 BGH, Urt. v. 26.1.1961 - II ZR 240/59, NJW 1961, 724, 2. Leitsatz: „Ist ein Gesellschafter wegen persönlicher Zahlungsschwierigkeiten bereit, im Interesse der Gesellschaft sofort auszuscheiden, und sind die anderen Gesellschafter damit einverstanden, so kann sich für einen minderiährigen Gesellschafter daraus die Rechtspflicht ergeben, dem Ausscheiden zuzustimmen, wenn er kein schutzwertes Interesse an dem Verbleiben gerade dieses Gesellschafters hat." 
Ausschließungsklage gegen einen Gesellschafter ${ }^{20}$ sowie zur Änderung von statutarischen Nachfolgeklauseln nach der Scheidung der Ehe der einer Kommanditgesellschaft angehörenden Eheleute ${ }^{21}$.

Die positive oder negative Stimmpflicht ist eine Primärfolge der Treuepflicht, die auf diese Weise in Natur durchgesetzt wird ${ }^{22}$. Sie wird von der Rechtsprechung jedoch nicht stets und vorbehaltlos bejaht, sondern nur unter gewissen Voraussetzungen. Insbesondere müsse, so der BGH, der Beschluss im Gesellschaftsinteresse dringend geboten und dem betroffenen Gesellschafter zumutbar $\operatorname{sein}^{23}$.

\section{Die Klagbarkeit des Wettbewerbsverbots von Geschäftsleitern in der Kapitalgesellschaft \& Co. KG}

Das zweite Beispiel betrifft das aus der organschaftlichen Treuepflicht folgende Wettbewerbsverbot von Geschäftsleitern ${ }^{24}$. Dessen Inhalt ist insbesondere in Dreieckskonstellationen, wie sie in der Kapitalgesellschaft \& Co. KG vorkommen, nicht unumstritten ${ }^{25}$. Die h.M. nimmt an, dass die Treuepflichten, denen die Geschäftsleiter der Komplementärgesellschaft unterliegen, nicht nur (unmittelbar) gegenüber der Komplementärgesellschaft wirken, sondern auch (mittelbar) Drittwirkung zugunsten der Kommanditgesellschaft entfalten, zu der die Geschäftsleiter keine organschaftlichen oder schuldrechtlichen Bezie-

$20 \mathrm{BGH}$, Urt. v. 28.4.1975 - II ZR 16/73, BGHZ 64, 253, 2. Leitsatz: „Bei Vorliegen eines Ausschließungsgrundes in der Person eines Gesellschafters kann der einzelne Gesellschafter auch ohne besondere gesellschaftsvertragliche Regelung verpflichtet sein, zu einer Ausschließungsklage seine Zustimmung zu geben." Hierzu Ulmer, JZ $1976,97 \mathrm{f}$

21 BGH, Urt. v. 18.3.1974 - II ZR 80/72, WM 1974, 831, 833.

22 Zum Grundsatz der Naturalerfüllung als einem beherrschenden Grundsatz des deutschen Privatrechts Weller, Die Vertragstreue, 2009, S. $42 \mathrm{ff} ., 316 \mathrm{ff} ., 371 \mathrm{ff}$.

23 BGH, Urt. v. 25.9.1986 - II ZR 262/85, BGHZ 98, 276, 280: „Die Treuepflicht kann einem Gesellschafter vielmehr gebieten, einer Anpassung des Gesellschaftsvertrages an veränderte Verhältnisse zuzustimmen, die mit Rücksicht auf das Gesellschaftsverhältnis, insbesondere zur Erhaltung des Geschaffenen, dringend geboten und den Gesellschaftern unter Berücksichtigung ihrer eigenen schutzwerten Belange zumutbar ist." Vgl. zu den Einschränkungen einer Stimmpflicht auch Hueck/Fastrich in Baumbach/Hueck, GmbHG, 19. Aufl. 2010, § 13 Rz. 29.

24 Zum Wettbewerbsverbot von GmbH-Geschäftsführern: BGH, Urt. v. 9.11.1967 II ZR 64/67, BGHZ 49, 30, 31 (obiter dictum); OLG Oldenburg, Urt. v. 17.2.2000 1 U 155/99, NZG 2000, 1038, 1039; Altmeppen in Roth/Altmeppen, GmbHG, 6. Aufl. 2009, §6 Rz. 78, 83 sowie § 43 Rz. 26, 29; Klöhn in Bork/Schäfer, GmbHG, 2010, § 43 Rz. 38; Zöllner/Noack in Baumbach/Hueck, GmbHG, 19. Aufl. 2010, § 35 Rz. 41; Riegger, BB 1983, 90. Zum Wettbewerbsverbot des Vorstands in der AG Fleischer, AG 2005, 336 ff.; Hüffer, AktG, 9. Aufl. 2010, §88 Rz. 1 sowie $§ 84$ Rz. 9; vgl. ferner Ziff. 4.3.1 DCGK.

25 Ausführlich Weller, ZHR 175 (2011), 110, 133 ff. 
hungen haben ${ }^{26}$. Begründet wird diese Drittwirkung nach überzeugender Auffassung mit der Figur des Vertrages mit Schutzwirkung zugunsten Dritter ${ }^{27}$.

Problematisch ist allerdings, mit welchen Rechtsfolgen diese drittschützenden Treuepflichten einhergehen. Eröffnen sie der Kommanditgesellschaft auf Primärebene einen klagbaren Anspruch auf Unterlassung von Wettbewerb oder gewähren sie ihr lediglich einen Anspruch auf Schadensersatz, wenn das Wettbewerbsverbot durch den Geschäftsleiter verletzt wird?

\section{a) Die Gruner + Jahr-Entscheidung des BGH}

Der BGH hat zu dieser Frage in der Gruner + Jahr-Entscheidung in einem obiter dictum Stellung genommen ${ }^{28}$. Zur Problemerhellung sei der Sachverhalt /vereinfacht) skizziert:

Wesentlicher Unternehmensträger des europaweit größten Druck- und Verlagsunternehmens Gruner + Jahr ist die Gruner + Jahr AG \& Co. KG. Diese hat drei Gesellschafter: zwei Kommanditisten und eine Komplementär-AG: Als Mehrheitskommanditistin die Bertelsmann AG (beteiligt mit ca. $74 \%$ ), als Minderheitskommanditist den Constanze Verlag (mit einer Beteiligung von ca. $24 \%$ ) und schließlich als Komplementärin die Gruner + Jahr AG (2\%).

An der Gruner + Jahr AG sind wiederum Bertelsmann (74,6\%) und der Constanze Verlag beteiligt $(25,1 \%)$. Es handelt sich also um eine "klassisch" strukturierte Kapitalgesellschaft \& Co. KG, bei der die Kommanditisten und AG-Gesellschafter personenidentisch sind und in der KG bzw. AG über den gleichen Einfluss verfügen.

Bekanntlich werden die Geschäfte in einer KG von der Komplementärin geführt, hier also von der Gruner + Jahr AG. Da diese AG durch ihre Organe handelt, leitet praktisch der Vorstand der AG die Geschäfte der Kommanditgesellschaft. Vor diesem Hintergrund stellt sich die Frage, ob die Vorstandsmitglieder der Komplementär-AG nicht nur der AG gegenüber, sondern auch unmittelbar gegenüber der KG einem Wettbewerbsverbot unterliegen. Diese Frage wurde virulent, als der Vorstandsvorsitzende X der Komplementär-AG zusätzlich in der Bertelsmann AG zum Vorstandsmitglied bestellt wurde. Es wurde also ein sog. Vorstandsdoppelmandat begründet, bei dem die Konkurrenzproblematik auf der Hand liegt: X sollte nicht nur als Organ der Komple-

26 Grundlegend BGH, Urt. v. 12.11.1979 - II ZR 174/77, BGHZ 75, 321, 322 f. (für Publikums-KGs); erweitert durch BGH, Urt. v. 24.3.1980 - II ZR 213/77, BGHZ 76, 326, 327, 337 f. (allgemein für $\mathrm{GmbH} \&$ Co. KGs); bestätigt durch BGH, Urt. v. 17.3.1987 VI ZR 282/85, BGHZ 100, 190, 193; Grunewald in MünchKomm. HGB, 2. Aufl. 2007, § $161 \mathrm{Rz} .82$ sowie $§ 165 \mathrm{Rz} .14$; Riegger, BB 1983, 90, 91.

27 Hüffer, AktG, 9. Aufl. 2010, 88 Rz. 4; Kort in Großkomm. AktG, 4. Aufl. 2006, 888 Rz. 47; Werner, GmbHR 2007, 988, 989.

28 BGH, Urt. v. 9.3.2009 - II ZR 170/07, NZG 2009, 744; hierzu Grigoleit, ZGR 2010, 662; Klöhn/Schaper, LMK 2009, 287721; s. auch die Entscheidung der Vorinstanz: OLG Hamburg, Urt. v. 29.6.2007 - 11 U 141/06, ZIP 2007, 1370; hierzu Altmeppen, ZIP 2008, 437; Cahn, Der Konzern 2007, 716; Hellgardt, ZIP 2007, 2248; Werner, GmbHR 2007, 988. 
mentär-AG die Geschäfte von Gruner + Jahr führen, sondern fortan zugleich auch die Geschäfte des Konkurrenzunternehmens Bertelsmann.

Diese Konkurrenzproblematik rief denn auch den Minderheitskommanditisten Constanze Verlag auf den Plan. Er begehrte mit seiner Klage die Feststellung, dass X aufgrund seiner organschaftlichen Treuepflicht auch gegenüber der Gruner + Jahr AG \& Co. KG einem Wettbewerbsverbot unterliege. X dürfe das zweite Vorstandsmandat in der Bertelsmann AG daher nur mit vorheriger Zustimmung der KG ausüben; anders gewendet: die KG habe ein Vetorecht im Hinblick auf die Begründung des Vorstandsdoppelmandates.

\section{b) BGH: Drittschutz nicht auf Primärebene (Unterlassung), sondern nur auf Sekundärebene (Schadensersatz)}

Der BGH weist dieses Klagebegehren zurück. Er lässt „dahinstehen, ob bei der AG \& Co. KG die Norm des $\S 88$ Abs. 1 Satz 2 AktG oder das Organ- und Anstellungsverhältnis des Vorstands zur Komplementär-AG tatsächlich drittschützende Wirkung zugunsten der KG entfalte[t], wie dies für den Geschäftsführer der $\mathrm{GmbH} \& \mathrm{Co}$. KG angenommen wird“29.

Der BGH kann diese Frage deshalb offenlassen, weil er der zum Wettbewerbsverbot verdichteten organschaftlichen Treuepflicht auf Primärebene die Klagbarkeit abspricht; er beschränkt die Rechtsfolgen der Treuepflicht auf die Sekundärebene in Form von Schadensersatz: „Denn Drittschutz bedeutet in diesem Zusammenhang nur, dass der KG eigene Ansprüche zustehen könnten, soweit der Geschäftsleiter (Treu-)Pflichten aus dem den Drittschutz begründenden, bereits bestehenden Anstellungs- und Organverhältnis zur Komplementärin verletzt" ${ }^{\prime 30}$. Dagegen sei ein Recht der KG-Gesellschafter „auf maßgebliche Mitwirkung (faktisches, Vetorecht') bei dem - zeitlich vorgehenden ,primären' Akt der Bestellung von Vorstandsmitgliedern zu Doppelmandatsträgern" mit dem Drittschutzkonzept nicht verbunden ${ }^{31}$.

Nach Auffassung des BGH soll das Wettbewerbsverbot für den Begünstigten also nur ex post, nicht auch ex ante wirken: Es soll keine Primäransprüche (Unterlassung), sondern nur Sekundäransprüche im Verletzungsfall hervorbringen, was auf ein "dulde und liquidiere" hinausläuft ${ }^{32}$. Eine dogmatisch stringente Begründung für seine Position liefert der BGH freilich nicht.

\section{Befund: Keine stringente Klagbarkeitsdogmatik bei gesellschaftsrechtlichen Treuepflichten}

Die Beispiele zur treuepflichtbasierten Stimmpflicht einerseits und zum treuepflichtbasierten Wettbewerbsverbot andererseits lassen bisher kein stimmiges System im Hinblick auf die Klagbarkeit von Treuepflichten im Gesellschafts-

29 BGH, Urt. v. 9.3.2009 - II ZR 170/07, NZG 2009, 744, Tz. 21.

30 BGH, Urt. v. 9.3.2009 - II ZR 170/07, NZG 2009, 744, Tz. 21.

31 BGH, Urt. v. 9.3.2009 - II ZR 170/07, NZG 2009, 744, Tz. 22.

32 Ähnliche Interpretation von Klöhn/Schaper, LMK 2009, 287721. 
recht erkennen. Unstreitig ist zwar, dass eine Treuepflichtverletzung auf Sekundärebene Schadensersatz auslösen kann; ob die Treuepflicht aber auch schon auf der vorgelagerten Primärebene in Natur durchgesetzt werden kann, wird momentan nur disparat und ohne erkennbares System von Fall zu Fall angenommen.

\section{These}

\section{Rekurs auf die allgemeine Klagbarkeitsdogmatik von Rücksichtspflichten}

Eine systemstimmige Lösung würde sich ergeben, wenn man im Hinblick auf die Frage nach der Klagbarkeit gesellschaftsrechtlicher Treuepflichten auf die allgemeine Klagbarkeitsdogmatik von Rücksichtspflichten rekurrierte. Rücksichtspflichten haben ihre normative Grundlage in $\S 241$ Abs. 2 BGB. Werden sie verletzt, hat der Schuldner nach $§ 280$ Abs. 1 BGB Schadensersatz zu leisten.

Im Unterschied zu den in $\S 241$ Abs. 1 BGB verankerten Leistungspflichten sind Rücksichtspflichten auf Primärebene nicht stets, sondern nur unter zwei Voraussetzungen in Natur durchsetzbar ${ }^{33}$ : (1.) Ihr Pflichtinhalt muss sich hinreichend konkretisieren lassen. (2.) Ferner muss ein überwiegendes schutzwürdiges Interesse desjenigen bestehen, der die Beachtung der Rücksichtspflicht einfordert.

\section{Begründung}

Die These, auf die allgemeine schuldrechtliche Klagbarkeitsdogmatik zurückzugreifen, um die Rechtsfolgenproblematik der Treuepflichten im Gesellschaftsrecht nach einheitlichen Voraussetzungen zu lösen, lässt sich in mehrfacher Hinsicht begründen. Zum einen ist die Systemstimmigkeit einer Lösung ein rechtlicher Wert an sich, der nicht zuletzt im Hinblick auf die Rechtssicherheit und Vorhersehbarkeit rechtlicher Entscheidungen wesentlich ist. Zum anderen würde nach dem hier zur Diskussion gestellten Vorschlag die gesellschaftsrechtliche Treuepflichtdogmatik mit der allgemeinen schuldrechtlichen Dogmatik subjektiver Rechte und Pflichten auch auf Rechtsfolgenebene verzahnt - eine Verzahnung, welche die h.M. im Zusammenhang mit der Rechtsnatur von Treuepflichten und der darauf basierenden Unterscheidung zwischen mitgliedschaftlichen und organschaftlichen Treuepflichten bereits vornimmt, wenn sie sich diesbezüglich auf die allgemeine Lehre von der Interessenstruktur von Verträgen stützt ${ }^{34}$. Schließlich harmonierte dieser Ansatz in normativer Hinsicht mit der Verwurzelung der Treuepflichtdogmatik in den $\S \S 241$ Abs. 2, 242 BGB. Die Treuepflichten gelten nämlich als ein Unterfall der Rücksichtspflichten gemäß § 241 Abs. 2 BGB. Demnach

33 Ausführlich zur Klagbarkeit von Leistungs- und Rücksichtspflichten Weller, Die Vertragstreue, 2009, S. $232 \mathrm{ff} ., 264 \mathrm{ff}$.

34 Hierzu infra unter IV.2. und 3. 
wäre es nur konsequent, wenn man sich hinsichtlich der Klagbarkeit der Treuepflichten an der im schuldrechtlichen Schrifttum elaborierten Klagbarkeitsdogmatik der Rücksichtspflichten orientierte.

Im Folgenden soll zunächst dargelegt werden, dass sich auch die gesellschaftsrechtliche Treuepflichtdogmatik normativ auf die $\S \S 241$ Abs. 2, 242 BGB zurückführen lässt (unter IV.). Darauf aufbauend wird die allgemeine Klagbarkeitsdogmatik von Rücksichtspflichten erörtert (unter V.), bevor auf ihrer Grundlage die beiden Eingangsbeispiele zur Klagbarkeit der gesellschaftsrechtlichen Treuepflicht (Stimmpflicht, Wettbewerbsverbot) gelöst werden (unter VI.).

\section{Normative Verankerung der Treuepflichtdogmatik in $\S \S 241$, 242 BGB}

\section{Schuldrechtliche Treuepflicht}

Die allgemeine schuldrechtliche Treuepflichtdogmatik beruht auf der Erkenntnis, dass jedes Vertragsverhältnis in Verbindung mit $\S 242$ BGB Treuepflichten begründet $^{35}$. So hebt der BGH hervor, mit jedem Vertrag sei „die aus dem Rechtsgrundsatz von Treu und Glauben (§ 242 BGB) folgende vertragliche Nebenpflicht verbunden, sich leistungstreu zu verhalten, also alles zu unterlassen, was den Vertragszweck gefährden oder vereiteln könnte, und alles Notwendige zu tun, um die Erfüllung der vertraglich übernommenen Verpflichtung sicherzustellen" ${ }^{\prime \prime 3}$. In ihrer negativen Wirkrichtung verpflichtet die Treuepflicht den Adressaten mithin zu einem Unterlassen von Handlungen, die den Vertragszweck konkret beeinträchtigen ${ }^{37}$. In ihrer positiven Wirkrichtung erlegt sie den Parteien auf, diejenigen Maßnahmen zu ergreifen, die erforderlich und zumutbar sind, um den Vertragszweck vorzubereiten, herbeizuführen und zu sichern ${ }^{38}$. Die Treuepflichten treten als vertraglich nicht ausdrücklich geregelte, jedoch aus $\S 242$ BGB ableitbare Rücksichtspflichten $(\S 241$ Abs. 2 $\mathrm{BGB})^{39}$ neben die vereinbarten Leistungspflichten (§ 241 Abs. 1 BGB) und sollen die Verwirklichung des Vertragszwecks in Natur (Naturalerfüllung) flan-

35 K. Schmidt, Gesellschaftsrecht, 4. Aufl. 2002, §20 IV 1; Weller, Die Vertragstreue, 2009, S. 302 ff., 309 ff.; M. Winter, Mitgliedschaftliche Treuebindungen im GmbHRecht, 1988, S. $10 \mathrm{ff}$.

36 BGH, Urt. v. 30.3.1995 - IX ZR 182/94, NJW 1995, 1954, 1955.

37 Gernhuber, Das Schuldverhältnis, 1989, S. 21 f.; Krebs, Sonderverbindung und außerdeliktische Schutzpflichten, 2000, S. 447 ff.; Wiedemann in Soergel, BGB, 1990, Vor $\S 275 \mathrm{Rz}$. 373. Die Treuepflichten halten die Parteien insbesondere dazu an, "den Vertragspartner nicht daran zu hindern, die von ihm mit dem Vertragsabschluss angestrebten Vorteile zu erlangen oder seine Ziele zu verwirklichen", Otto in Staudinger, BGB, 2004, § $282 \mathrm{Rz} .35$.

38 Gernhuber, Das Schuldverhältnis, 1989, S. 22; Krebs, Sonderverbindung und außerdeliktische Schutzpflichten, 2000, S. 447 ff.; Roth in MünchKomm. BGB, 5. Aufl. 2007, § $242 \mathrm{Rz} .146$.

39 Rechtsdogmatisch ist die Treuepflicht den Rücksichtspflichten zuzuordnen, die ihre normative Grundlage seit der Schuldrechtsreform 2001 in den $\S \S 242,241$ Abs. 2 BGB haben, Roth/Weller, Handels- und Gesellschaftsrecht, 7. Aufl. 2010, Rz. 288. 
kierend sichern. Dabei ist das inhaltlich aus der Treuepflicht folgende Pflichtenprogramm vertrags- und situationsabhängig: Es orientiert sich am Vertragszweck und den jeweils für die Vertragserfüllung relevanten Umständen ${ }^{40}$.

\section{Mitgliedschaftliche Treuepflicht}

Die Treuepflicht des Gesellschafters (mitgliedschaftliche Treuepflicht) besteht sowohl im Verhältnis zur Gesellschaft als auch im Verhältnis zu den Mitgesellschaftern ${ }^{41}$. Sie hat ihre Wurzel nach überzeugender Auffassung kumulativ im Gesellschaftsvertrag ${ }^{42}$ (mit seiner Doppelnatur als Schuld- und Organisationsvertrag) und in $\S 242$ BGB $^{43}$. Der Bezug zum Gesellschaftsvertrag liegt darin, dass die Treuepflicht die darin übernommene Förderpflicht des Gesellschafters flankierend sichert ${ }^{44}$. Letztere erschöpft sich nicht nur in der Leistung der unmittelbar versprochenen Beiträge (§ $706 \mathrm{BGB})$, sondern beinhaltet darüber hinausgehend eine fortwährende Verpflichtung des Gesellschafters, sein Handeln am gemeinsamen Vertragszweck auszurichten, zu dessen Verwirklichung beizutragen und sich für das gemeinsame Ziel einzusetzen ${ }^{45}$. Die Treuepflicht verlangt diesbezüglich, dass die Art und Weise dieses Handelns „gesellschaftstreu" erfolgt und alles unterlassen wird, was die Erreichung des Gesellschaftszwecks hindern oder gefährden könnte ${ }^{46}$. Dabei ist zu berücksichtigen, dass es sich bei Gesellschaftsverträgen „typischerweise um langfristige, ,nach vorne offene' und deshalb notwendig unvollständige Verträge ${ }^{\prime \prime 47}$ handelt. Bei diesen bedürfen die vertraglich ausdrücklich vereinbarten Pflichten notwendigerweise einer situationsabhängigen Ergänzung durch ungeschriebene, hinreichend flexible Pflichten, mit denen sich gesellschaftsvertraglich nicht geregelte Konfliktlagen lösen lassen ${ }^{48}$. Da sich diese letzteren Pflichten, die Treuepflichten, aufgrund ihrer erst nachträglichen, situationsgebun-

40 BGH, Urt. v. 30.3.1995 - IX ZR 182/94, NJW 1995, 1954, 1955; M. Winter, Mitgliedschaftliche Treuebindungen im GmbH-Recht, 1988, S. 14 f.

41 K. Schmidt, Gesellschaftsrecht, 4. Aufl. 2002, §20 IV 1 c); Weller in Bork/Schäfer, GmbHG, 2010, § $13 \mathrm{Rz} .7$.

42 Eindrücklich zur gesellschaftsvertraglichen Grundlage BGH, Urt. v. 6.12.1962 - KZR 4/62, BGHZ 38, 306, 309, 311, 316 (für das Wettbewerbsverbot als Ausprägung der Treuepflicht). Die mitgliedschaftliche Treuepflicht gilt unabhängig davon, ob der Gesellschafter bei der Gründung mitgewirkt hat oder erst später durch Beitritt oder Anteilsübertragung an den Gesellschaftsvertrag gebunden wurde, Wiedemann, WM 2009, 1, 4.

43 M. Winter, Mitgliedschaftliche Treuebindungen im GmbH-Recht, 1988, S. 12 ff.; vgl. zu weiteren Ansätzen Weller in Bork/Schäfer, GmbHG, 2010, §13 Rz. 7.

44 Goette in Ebenroth/Boujong/Joost/Strohn, HGB, 2. Aufl. 2008, §112 Rz. 1; M. Winter, Mitgliedschaftliche Treuebindungen im GmbH-Recht, 1988, S. $14 \mathrm{f}$.

45 BGH, Urt. v. 21.2.1978 - KZR 6/77, BGHZ 70, 331, 335; M. Winter, Mitgliedschaftliche Treuebindungen im GmbH-Recht, 1988, S. 14.

46 BGH, Urt. v. 6.12.1962 - KZR 4/62, BGHZ 38, 306, 312; Armbrüster, ZIP 1997, 261 , 266; Goette in Ebenroth/Boujong/Joost/Strohn, HGB, 2. Aufl. 2008, § 112 Rz. 1.

47 Verse, Treuepflichten und Gleichbehandlungsgrundsatz, in Bayer/Habersack (Hrsg.), Aktienrecht im Wandel, Bd. II, 2007, S. 579, 582.

48 Verse, Treuepflichten und Gleichbehandlungsgrundsatz, in Bayer/Habersack (Hrsg.), Aktienrecht im Wandel, Bd. II, 2007, S. 579, 582. 
denen Konkretisierung jedoch regelmäßig nicht auf den tatsächlich bei Vertragsschluss geäußerten Willen der Vertragsschließenden zurückführen und sich damit nicht allein aus dem Gesellschaftsvertrag ableiten lassen, bedarf es für ihre Begründung zusätzlich des Rückgriffs auf $\S 242 \mathrm{BGB}^{49}$.

\section{Organschaftliche Treuepflicht}

Von der mitgliedschaftlichen Treuepflicht der Gesellschafter ist die sog. organschaftliche Treuepflicht der Geschäftsleiter zu unterscheiden ${ }^{50}$. Jene fungiert nach der auf Zöllner ${ }^{51}$ zurückgehenden Lehre von der Einwirkungsmacht als Korrelat der weitreichenden Befugnisse der Geschäftsleiter im Hinblick auf die Verwaltung des ihnen anvertrauten Vermögens sowie ihres Informationsvorsprungs im Hinblick auf Geschäftsinterna ${ }^{52}$. Als dogmatische Grundlage kommen zwei Rechtsverhältnisse in Betracht, die zwischen dem Geschäftsleiter und der Komplementär-Kapitalgesellschaft bestehen: das durch Bestellung begründete organschaftliche Verhältnis einerseits und der schuldrechtliche Anstellungsvertrag andererseits ${ }^{53}$, jeweils in Kombination mit $\S 242$ BGB $^{54}$. Ob die Treuepflicht aus dem Anstellungsvertrag ${ }^{55}$, aus dem Organverhältnis ${ }^{56}$ oder aber aus beiden Rechtsverhältnissen ${ }^{57}$ folgt, ist für die vorliegenden Zwecke unerheblich, da die Treuepflicht aus dem Anstellungsvertrag mit derjenigen aus dem Organverhältnis inhaltlich kongruent geht ${ }^{58}$.

\section{Zwischenbilanz}

Als Zwischenbilanz lässt sich festhalten, dass die gesellschaftsrechtlichen (d.h. die mitgliedschaftlichen und die organschaftlichen) Treuepflichten ebenso wie die schuldrechtlichen Treuepflichten auf die jeweilige vertragliche Sonderbeziehung in Kombination mit dem Grundsatz von Treu und Glauben

49 M. Winter, Mitgliedschaftliche Treuebindungen im GmbH-Recht, 1988, S. 15.

50 Fleischer, WM 2003, 1045, 1046 f.; Hüffer, AktG, 9. Aufl. 2010, §84 Rz. 9; Wiedemann, WM 2009, $1 \mathrm{ff}$.

51 Zöllner, Die Schranken mitgliedschaftlicher Stimmrechtsmacht bei den privatrechtlichen Personenverbänden, 1963, S. 342 f.

52 Zöllner/Noack in Baumbach/Hueck, GmbHG, 19. Aufl. 2010, § 35 Rz. 39; Altmeppen in Roth/Altmeppen, GmbHG, 6. Aufl. 2009, §43 Rz. 26.

53 Fleischer, WM 2003, 1045, 1046; Zöllner/Noack in Baumbach/Hueck, GmbHG, 19. Aufl. 2010, § $35 \mathrm{Rz} .12$ ff., 16 und $163 \mathrm{ff}$.

54 Vgl. auch Altmeppen, ZIP 2008, 437, 440: „Dogmatische Grundlage i. S.d. Rechtsquelle ist letztlich $\S 242$ BGB."

55 OLG Köln, Urt. v. 10.1.2008 - 18 U 1/07, NZG 2009, 306, 307; Goette, Die GmbH, 2. Aufl. 2002, §8 Rz. $142 \mathrm{ff}$. (für das Wettbewerbsverbot); Grunewald in MünchKomm. HGB, 2. Aufl. 2007, § $161 \mathrm{Rz} .82$.

56 OLG Oldenburg, Urt. v. 17.2.2000 - 1 U 155/99, NZG 2000, 1038, 1039; Hüffer, AktG, 9. Aufl. 2010, § 84 Rz. 9; vgl. auch OLG Hamburg, Urt. v. 29.6.2007-11 U 141/06, ZIP 2007, 1370, 1372.

57 Altmeppen, ZIP 2008, 437, 440; Grunewald in MünchKomm. HGB, 2. Aufl. 2007, $\S 165 \mathrm{Rz} .14$.

58 Fleischer, WM 2003, 1045, 1046; Zöllner/Noack in Baumbach/Hueck, GmbHG, 19. Aufl. 2010, § $35 \mathrm{Rz} .39$. 
gestützt werden. Ihr Pflichteninhalt ist auf den flankierenden Schutz des Vertragszwecks gerichtet /Verwirklichung des Gesellschaftszwecks bzw. des Zwecks sorgfältiger Geschäftsführung).

Da es sich bei den Treuepflichten im Unterschied zu den Beitrags- und Geschäftsführungspflichten nicht um Leistungspflichten (§241 Abs. 1 BGB) handelt, sind sie den Rücksichtspflichten ( $\$ 241$ Abs. 2 BGB) zuzuordnen. Denn nach vorzugswürdiger Meinung kennt das deutsche Privatrecht über Leistungsund Rücksichtspflichten hinaus keine dritte Pflichtenkategorie, jedenfalls nicht seit der Neufassung des $\S 241$ BGB im Zuge der Schuldrechtsreform $(2001)^{59}$. Normative Grundlage der gesellschaftsrechtlichen Treuepflichten sind mithin die $\S \S 241$ Abs. 2, 242 BGB.

\section{Die Klagbarkeit von Rücksichtspflichten (§ 241 Abs. 2 BGB)}

Wenn aber die gesellschaftsrechtlichen Treuepflichten ihre normative Grundlage in den $\S \S 241$ Abs. 2, 242 BGB haben, erscheint es konsequent, auf jene die zu diesen Bestimmungen entwickelte allgemeine Klagbarkeitsdogmatik zu übertragen. Vor diesem Hintergrund soll nachfolgend zunächst der Meinungsstand zur Klagbarkeit von Rücksichtspflichten skizziert werden.

\section{Die Einzelelemente eines Schuldverhältnisses}

Ein Schuldverhältnis besteht in funktionaler Hinsicht nach moderner Doktrin aus mehreren Einzelelementen. Diese stellen sich aus der Sicht des Gläubigers als Einzelbefugnisse und aus Sicht des Schuldners als Einzelgebote dar. Nach Jürgen Schmidt sind insbesondere folgende Einzelbefugnisse eines Forderungsrechtes zu unterscheiden ${ }^{60}$ : Die Einziehungsbefugnis, die Klagebefugnis, die Vollstreckungsbefugnis, die Aufrechnungsbefugnis, die Verfügungsbefugnis und die Befugnis zum Behaltendürfen, die den bereicherungsrechtlichen Rechtsgrund bildet.

\section{Die Klagbarkeit als Eigenschaft von Leistungspflichten}

Von der Eigenschaft der Klagebefugnis hängt ab, ob ein subjektives Recht (in Rechtssystemen mit staatlichem Gewaltmonopol) gerichtlich als solches durchgesetzt werden kann. Die Klagebefugnis wird seit Bernhard. Windscheids

59 Weller, Die Vertragstreue, 2009, S. 242 ff., 252 ff. (str.).

$60 \mathrm{~J}$. Schmidt in Staudinger, BGB, 1995, Einl. zu $\S \S 241 \mathrm{ff} . \mathrm{Rz} .118 \mathrm{ff} . ; \mathrm{vgl}$. ferner die Anspruchsanalyse bei Schulze, Die Naturalobligation - Rechtsfigur und Instrument des Rechtsverkehrs einst und heute - zugleich Grundlegung einer zivilrechtlichen Forderungslehre, 2008, S. 461 ff., sowie Neumann, Leistungsbezogene Verhaltenspflichten - Zur Durchsetzung sogenannter vertraglicher Nebenpflichten, 1989, S. 19 ff.; in der älteren Literatur ist diesbezüglich insbesondere hervorzuheben Siber, Der Rechtszwang im Schuldverhältnis nach deutschem Reichsrecht, 1903, S. 17 ff., $68 \mathrm{ff}$. 
bahnbrechenden Arbeiten zur actio ${ }^{61}$ im deutschen Recht als materiellrechtliche Eigenschaft von Forderungsrechten angesehen; sie ist diesen grundsätzlich immanent ${ }^{62}$. Dementsprechend sind auch die den Forderungsrechten korrespondierenden ${ }^{63}$ Leistungspflichten klagbar, d.h., sie können in Natur vor Gericht geltend gemacht und im Vollstreckungswege durchgesetzt werden ${ }^{64}$. Der Gesetzgeber des BGB hat sich die Sichtweise von der Klagbarkeit von Forderungsrechten und Leistungspflichten zu Eigen gemacht und sie in $\S 241$ Abs. 1 BGB normativ verankert ${ }^{65}$.

\section{Die Klagbarkeit als Eigenschaft von Rücksichtspflichten?}

Im Unterschied zu den Leistungspflichten besteht für die Rücksichtspflichten keine entsprechende normative Verankerung, wurden sie doch erst nach Inkrafttreten des BGB "entdeckt". Ihre Kodifizierung in $\S 241$ Abs. 2 BGB im Zuge der Schuldrechtsreform 2001 hat die Frage nach ihrer Klagbarkeit unberührt gelassen. Dementsprechend kontrovers wird beurteilt, ob sie selbständig, d.h. unabhängig von der Geltendmachung einer Leistungspflicht, in Natur klagbar sind ${ }^{66}$.

\section{a) Entwicklung und Meinungsstand}

Die Problematik der Klagbarkeit von Treuepflichten dreht sich darum, ob jene nur verletzbar sind und sich insofern in Schadensersatzansprüchen $1 \$ 280$ Abs. 1 BGB) erschöpfen ${ }^{67}$, oder ob und inwiefern der Begünstigte bei drohender oder tatsächlicher Missachtung der Treuepflicht deren Einhaltung auch selbständig vor Gericht einklagen und im Vollstreckungswege durchsetzen kann ${ }^{68}$.

61 Windscheid, Die Actio des römischen Zivilrechts vom Standpunkte des heutigen Rechts, 1856; ders., Die Actio - Abwehr gegen Dr. Theodor Muther, 1857.

62 Weller, Die Vertragstreue, 2009, S. $232 \mathrm{ff}$.

63 Zur Korrespondenztheorie Weller, Die Vertragstreue, 2009, S. 223 ff.

64 Weller, Die Vertragstreue, 2009, S. 264.

65 Weller, Die Vertragstreue, 2009, S. $381 \mathrm{ff}$.

66 Vgl. zum Streitstand Neumann (Fn. 60), S. 14 ff.; J. Schmidt in Staudinger, BGB, 1995, Einl. zu $\S \S 241 \mathrm{ff} . \mathrm{Rz} .322 \mathrm{ff}$.; Kramer in MünchKomm. BGB, 5. Aufl. 2007, $\S 241 \mathrm{Rz} .12$; Roth in MünchKomm. BGB, 5. Aufl. 2007, § $242 \mathrm{Rz} .42$ ff., 64, 113; Olzen in Staudinger, BGB, 2009, §241 Rz. $544 \mathrm{ff}$.; Heinrichs in Palandt, BGB, 69. Aufl. 2009, §242 Rz. 25; Grigoleit in FS Canaris, 2007, Bd. I, S. 275, 291 f.; Köhler, AcP 190 (1990), 496, 503 ff.

67 So für die schuldrechtlichen Treuepflichten RG, Beschl. der Vereinigten Zivilsenate v. 24.1.1910 - I 188/08, RGZ 72, 393, 394: „(..) unser Recht kennt bei den auf ein Tun gerichteten Schuldverbindlichkeiten keinen klagbaren und ... vollstreckbaren Anspruch auf ein Unterlassen des mit der Verpflichtung zum Tun Unvereinbaren." Der Prinzipal habe aber u. a. einen Anspruch auf Schadensersatz. Vgl. ferner Krebs, Sonderverbindung und außerdeliktische Schutzpflichten, 2000, S. $547 \mathrm{ff}$.

68 Motzer, IZ 1983, 884, 886 f.; T. Schmidt in Staudinger, BGB, 1995, Einl: zu $§ \S 241 \mathrm{ff}$. Rz. 322. 


\section{aa) Die Doktrin Lehmanns und Sibers}

Die Klagbarkeit der heute sog. Rücksichtspflichten wurde lange Zeit im Kontext der Arbeiten von Lehmann und Siber gesehen. In seiner Habilitationsschrift „Die Unterlassungspflicht im Bürgerlichen Recht" aus dem Jahr 1906 unterscheidet Lehmann zwischen primären und sekundären Unterlassungspflichten. Die primären Unterlassungspflichten seien selbstständiger Gegenstand einer Leistungspflicht, die sekundären Unterlassungspflichten demgegenüber lediglich die Kehrseite positiver Verbindlichkeiten ${ }^{69}$. Lehmann legt hier die Grundlage für die später von Heinrich Stoll vorgenommene Unterscheidung zwischen Leistungspflichten und Schutzpflichten (letztere gehören heute zu den Rücksichtspflichten nach $\S 241$ Abs. 2 BGB ${ }^{70}$. Die von Lehmann so genannten primären Unterlassungspflichten sind in $\S 241$ Abs. 1 Satz 2 BGB erwähnt; demgegenüber sind die sekundären Unterlassungspflichten nach moderner Dogmatik ungeschriebener Teil der Rücksichtspflichten: Soweit es um den Integritätsschutz geht, erlegen die Rücksichtspflichten in ihrer Ausprägung als Schutzpflichten den Parteien die Pflicht auf, alles zu unterlassen, was die Rechtsgüter des Vertragspartners verletzen könnte. Soweit es um $\ddot{A}$ quivalenzschutz geht, erlegen die Rücksichtspflichten in Form der Treuepflichten beiden Vertragspartnern die Pflicht auf, alles zu unterlassen, was die Erreichung des Vertragszwecks gefährden oder vereiteln könnte.

Lehmann knüpfte an die Unterscheidung zwischen primären und sekundären Unterlassungspflichten unterschiedliche Rechtsfolgen: Während primäre Unterlassungspflichten selbständig eingeklagt werden könnten, seien sekundäre Unterlassungspflichten als solche nicht klagbar. Sie könnten nur entweder indirekt über die positive Leistungspflicht durchgesetzt oder im Verletzungsfall über die Geltendmachung von Schadensersatz liquidiert werden. Der BGH folgt in der Gruner + Jahr-Entscheidung ${ }^{71}$ unausgesprochen der Lehmannschen Doktrin und lehnt die selbständige Klagbarkeit im Hinblick auf die Unterlassung von Wettbewerb durch den Vorstand ab.

Bedeutung für die Klagbarkeitsproblematik hatte ferner die Lehre Sibers ${ }^{72}$. Ihm zufolge wird der Schuldner im Regelfall nur am Leistungserfolg gemessen, nicht aber an der Art und Weise, wie er diesen Leistungserfolg erreicht: „Die Verpflichtung zur konkreten Leistung (..) geht (...) auf weit mehr, als eine Pflicht zum sorgfältigen Versuch; sie geht auch auf die Herstellung eines Erfolges, für den die Anwendung der zu vertretenden Sorgfalt nicht ausreicht" ${ }^{\prime 73}$.

69 Lehmann, Die Unterlassungspflicht, 1906, 11 f., 166 ff.: „Die Pflicht zur Unterlassung der Zuwiderhandlung gegen den Zweck des Leistungsgebots ist nichts anderes wie der nach der negativen Seite hin ausgedachte Inhalt des Leistungsgebotes."

70 Hierzu Weller, Die Vertragstreue, 2009, S. 237 ff.

71 S. supra II.2.

72 Siber (Fn. 60), S. 175 ff.; Siber in Planck, BGB, 1914, Vorbem. vor § 241, III B.2.: „Die Schuld ist daher nicht erfüllt, wenn der Erfolg nicht eingetreten ist, mag auch der Schuldner alles von seiner Seite Erforderliche getan haben. (...) Die Schuld kann umgekehrt erfüllt sein, ohne dass der Schuldner das mindeste dazu getan hat, z. B. durch Leistung eines Dritten (§ 267)."

73 Siber (Fn. 60), S. 176 f. 
Weil der Schuldner hiernach keine Kraftanstrengung ${ }^{74}$ schuldet, soll ein bestimmtes Leistungsverwirklichungsverhalten auch nicht unmittelbar (selbständig), sondern nur mittelbar (unselbständig) über die Geltendmachung des geschuldeten Leistungserfolges eingefordert werden können ${ }^{75}$.

\section{bb) Position der Rechtsprechung}

Soweit (nicht vertraglich vereinbarte) leistungsbezogene Rücksichtspflichten in Frage stehen, die auf ein Unterlassen gerichtet sind, lehnt die Rechtsprechung - der Auffassung Lehmanns folgend - eine selbständige Klagbarkeit $a b^{76}$. Auf ein Unterlassen zielende (schuldrechtliche) Treuepflichten seien das bloße Kehrbild einer positiven Leistungspflicht und daher notwendig in dieser mitenthalten. Beispielsweise soll laut OLG Frankfurt der Lieferant von Gas gegenüber seinem Abnehmer, der sich verpflichtet hat, seinen gesamten Gasbedarf bei ihm zu decken, keinen aus der Leistungstreuepflicht folgenden klagbaren Anspruch auf Unterlassung anderweitigen Gasbezuges haben ${ }^{77}$.

\section{cc) Literatur: Bejahung der Klagbarkeit unter besonderen Voraussetzungen}

Die heute herrschende Literatur bejaht die selbständige Klagbarkeit von Rücksichtspflichten - sofern gewisse Voraussetzungen vorliegen (dazu sogleich) unabhängig davon, ob diese auf ein Tun oder ein Unterlassen gerichtet $\operatorname{sind}^{78}$.

Dem Klagbarkeits-Ansatz der Literatur ist zu folgen. Er wird bestätigt durch die Existenz einiger gesetzlich normierter Rücksichtspflichten, deren Klagbarkeit unstreitig ist, so etwa der Pflichten in $\S \S 535$ Abs. 1 Satz 2, 469, 541, 666 $\mathrm{BGB}$ und $\S 60 \mathrm{HGB}^{79}$. Darüber hinaus blickt die heutige Dogmatik in einer

74 Nach der früher vertretenen Kraftanstrengungslehre ist kein Erfolg, sondern nur „die Tätigkeit, die den Erfolg herbeizuführen geeignet ist", geschuldet. Diese Lehre konnte sich jedoch nicht durchsetzen, vgl. Jakobs, Unmöglichkeit und Nichterfüllung, 1969, S. $214 \mathrm{ff}$.

75 Vgl. RG, Beschl. der Vereinigten Zivilsenate v. 24.1.1910 - I 188/08, RGZ 72, 393 f. (s. auch die RG-Zitate in Fn. 67 und 76); vgl. auch Köhler, AcP 190 (1990), 496, 501, 503 ff.; Lenzen, NJW 1967, $1260 \mathrm{f}$.

76 OLG Frankfurt a. M., Urt. v. 26.4.1984 - 8 U 15/84, IZ 1985, 337; RG, Urt. v. 22.5.1931 - II 402/30, RGZ 133, 51, 62; RG, Beschl. der Vereinigten Zivilsenate v. 24.1.1910 - I 188/08, RGZ 72, 393, 394: „(..) jede Verpflichtung zu einem positiven Tun [trägt] die selbstverständliche Verbindlichkeit in sich, alles mit diesem positiven Tun Unvereinbare zu unterlassen. Aber diese negative Seite der Verpflichtung zum positiven Tun ist nicht der Inhalt der Leistung i. S.d. § 241 [Abs. 1] BGB."; vgl. auch Köhler, AcP 190 (1990), 496, 501, 503 ff.; Lenzen, NJW 1967, 1260 f.

77 OLG Frankfurt a. M., Urt. v. 26.4.1984-8 U 15/84, IZ 1985, 337.

78 Neumann (Fn. 60), S. 15 ff.; Schlechtriem/Schmidt-Kessel, Schuldrecht AT, 6. Aufl. 2005, Rz. 468; Mansel in Jauernig, BGB, 13. Aufl. 2009, § 241 Rz. 10; Olzen in Staudinger, BGB, 2005, § $241 \mathrm{Rz} .546 \mathrm{ff} . ;$ I. Schmidt in Staudinger, BGB, 1995, Einl. zu $\S \S 241$ ff. Rz. 324 f.; Grigoleit in FS Canaris, 2007, S. 275, 279, 290, 292; Lenzen, NIW 1967, 1260, 1261; Motzer, JZ 1983, 884, 886 f.; Stürner, IZ 1976, 384 f., 388 ff.; vgl. zur Sicherung von „Unterlassungsnebenansprüchen" durch einstweilige Verfügung nach § 938 ZPO Tauernig, ZZP 79 (1966), 321, $328 \mathrm{ff}$.

79 Schlechtriem/Schmidt-Kessel, Schuldrecht AT, 6. Aufl. 2005, Rz. 468. 
maßgeblich von Wieacker ${ }^{80}$ angestoßenen Fortentwicklung der Auffassung Sibers nicht mehr ausschließlich auf den Leistungserfolg als das geschuldete Ergebnis eines Vertrages, sondern auch auf die Pflichtgemäßheit des dahin führenden Leistungsverhaltens des Schuldners ${ }^{81}$ :

„Leistung ist nicht nur Leistungsverhalten des Schuldners, aber auch nicht nur Eintritt des Leistungserfolges, sondern Eintritt des Leistungserfolges durch ein Leistungsverhalten des Schuldners ${ }^{\prime \prime 2}$.

Das korrekte Leistungsverhalten gilt nunmehr als echte Pflicht, nicht mehr nur als negative Tatbestandsvoraussetzung eines Schadensersatzanspruchs ${ }^{83}$. Schließlich strahlt die Grundentscheidung der Rechtsordnung hinsichtlich des Vorrangs der Natural- vor der Pekuniarerfüllung ${ }^{84}$ auch positiv auf die Durchsetzbarkeit leistungsbezogener Rücksichtspflichten aus. Rolf Stürner formuliert hierzu treffend:

„Die Verweigerung des Anspruchs auf Erfüllung von Nebenpflichten wäre im Ergebnis nichts anderes als das Festhalten am Prinzip der Geldkondemnation für einen bestimmten Pflichtenkreis ${ }^{\prime \prime 85}$.

\section{b) Voraussetzungen der Klagbarkeit}

Über die Voraussetzungen, unter denen die Klagbarkeit leistungsbezogener Rücksichtspflichten bejaht werden kann, besteht in der Literatur - wenn auch nicht im Detail, so doch im Wesentlichen - Einigkeit:

\section{aa) Konkretisierbarkeit des Pflichtinhalts}

Die Klagbarkeit setzt zunächst voraus, dass die leistungsbezogene Rücksichtspflicht im Moment der Geltendmachung konkretisierbar ist; das eingeforderte Verhalten muss ex ante fassbar sein, und nicht erst ex post, wie es im Rahmen einer Schadensersatzwürdigung geschieht ${ }^{86}$. Denn die Bestimmtheit des Pflichtgegenstandes ist nicht nur von prozessualer Bedeutung für die Formulierung des Klageantrags ( $\$ 253 \mathrm{ZPO}$ ), der titulier- und vollstreckbar sein muss ${ }^{87}$, sondern auch eine ungeschriebene Voraussetzung der materiellrechtlichen Klagebefugnis ${ }^{88}$. An der Konkretisierbarkeit kann es beispielsweise fehlen, wenn noch nicht klar ist, welche von mehreren in Betracht kommenden Handlungsalternativen geeignet ist, die Vertragszweckrealisierung zu ermöglichen ${ }^{89}$.

80 Wieacker in FS Nipperdey, 1965, S. 783 ff., $798 \mathrm{ff}$.

81 Neumann (Fn. 60), S. 71; Stürner, JZ 1976, 384, 390.

82 Wieacker in FS Nipperdey, 1965, S. 783, 812.

83 Stürner, IZ 1976, 384, 390.

84 Ausführlich hierzu Weller, Die Vertragstreue, 2009, S. $316 \mathrm{ff}$.

85 Stürner, IZ 1976, 384, 389.

86 Grigoleit in FS Canaris, 2007, Bd. I, S. 275, 289 ff.; Motzer, IZ 1983, 884, 886; Stürner, IZ 1976, 384, 385; Olzen in Staudinger, BGB, 2005, § $241 \mathrm{Rz} .547$.

87 Stürner, JZ 1976, 384, 388.

88 Neumann (Fn. 60), S. 109 ff.; vgl. ferner Köhler, AcP 190 (1990), 496, 503, 509 f.

89 Vgl. auch Stürner, JZ 1976, 384, 386, 388. 
Neumann hat eine überzeugende Methode zur Konkretisierung leistungsbezogener Rücksichtspflichten herausgearbeitet ${ }^{90}$. Als Richtschnur kann u.a. auf die verkehrserforderliche Sorgfalt (§276 Abs. 1 Satz 2 BGB $)^{91}$ sowie auf die Prinzipien der objektiven Eignung und des subjektiven Könnens eines Verhaltens zur Erreichung des Leistungserfolges ${ }^{92}$ rekurriert werden. Kommen verschiedene Handlungen bzw. Unterlassungen in Frage, gibt die Verkehrsüblichkeit $\mathrm{Maß}^{93}$.

\section{bb) Überwiegendes schutzwürdiges Interesse}

Die Durchsetzung leistungsbezogener Rücksichtspflichten kommt entgegen Motzer $^{94}$ auch bei Konkretisierbarkeit des Pflichtinhalts nicht uneingeschränkt, sondern nur bei Vorliegen eines überwiegenden schutzwürdigen Interesses in Betracht ${ }^{95}$, weil sie die Handlungsfreiheit der involvierten Vertragsparteien, die in der Wahl der für die Vertragserfüllung erforderlichen Mittel eigentlich frei sein sollen, beschneidet ${ }^{96}$.

Allerdings dürfen die Anforderungen an dieses Interesse nicht überspannt werden. Abzulehnen ist daher die Voraussetzung eines „besonderen Präventionsinteresses", welches nur dann vorliegen soll, wenn existenzgefährdende Schäden oder die Verletzung von Rechtsgütern i. S.d. §823 Abs. 1 BGB drohen ${ }^{97}$. Das besondere Präventionsinteresse mag für die Klagbarkeit der klassischen Schutzpflichten sinnvoll sein; für die Klagbarkeit von Treuepflichten ist es jedoch nicht geeignet, weil diese den Schutz absoluter Rechtsgüter überhaupt nicht bezwecken.

Ausreichend für das Überspringen der ultima-ratio-Hürde ist - nach einer Einzelfallabwägung der widerstreitenden Parteiinteressen, wobei in die Abwägung nicht nur bestimmte Rechtsgüter, sondern alle schutzwürdigen Interessen der Beteiligten einzufließen haben - ein überwiegendes schutzwürdiges Interesse desjenigen, der die Beachtung der Rücksichtspflicht reklamiert ${ }^{98}$. Bei der Interessenabwägung kann man als Kontrollüberlegung mit Stürner berücksichtigen, ob die Verletzung der Rücksichtspflicht ohne Annahme einer Klagebefugnis durch anderweitige Rechtsfolgen (z.B. Schadensersatz) vollständig kompensiert werden kann, der Begünstigte also ohne Klagebefugnis nicht schlech-

90 Neumann, Leistungsbezogene Verhaltenspflichten, S. $109 \mathrm{ff}$.

91 Neumann, Leistungsbezogene Verhaltenspflichten, S. 116.

92 Neumann, Leistungsbezogene Verhaltenspflichten, S. 121 ff., 131.

93 Stürner, JZ 1976, 384, 388

94 Motzer, IZ 1983, 884, 886 f., der die Klagbarkeit von Schutzpflichten allein von ihrer Konkretisierbarkeit abhängig macht.

95 Köhler, AcP 190 (1990), 496, 508.

96 Krebs, Sonderverbindung und außerdeliktische Schutzpflichten, 2000, S. 547 f.

97 So Krebs, Sonderverbindung und außerdeliktische Schutzpflichten, 2000, S. 549 ff.; Olzen in Staudinger, BGB, 2005, § 241 Rz. 547; Grigoleit in FS Canaris, 2007, Bd. I, S. $275,290 \mathrm{f}$.

98 Neumann, Leistungsbezogene Verhaltenspflichten, S. 132 f.; Stürner, IZ 1976, 384, 389 ff.; vgl. auch Köhler, AcP 190 (1990), 496, 506 ff. für auf Unterlassung gerichtete Leistungstreuepflichten. 
ter stünde als mit ihrr9. Dies läuft auf die Faustformel hinaus, die Klagebefugnis bei solchen Interessen zu versagen, die im Verletzungsfall vollständig in Geld ausgeglichen werden können. Umgekehrt spricht viel für ein überwiegendes schutzwürdiges Interesse an der Klagbarkeit einer Rücksichtspflicht, wenn ihre Verletzung nur unzulänglich in Geld kompensiert werden könnte, etwa wenn immaterielle Interessen im Spiel sind oder der Schaden praktisch nur schwer nachweisbar ist.

Schließlich ist bei Rücksichtspflichten, die auf ein Unterlassen gerichtet sind, die Bejahung der Klagbarkeit entsprechend der allgemeinen Unterlassungspflichtdogmatik an das Vorliegen einer Verletzungs- oder Begehungsgefahr geknüpft, wobei eine Erstbegehungsgefahr ausreicht ${ }^{100}$.

\section{Lösung der Eingangsbeispiele}

Überträgt man nach der hier vertretenen These die allgemein für Rücksichtspflichten geltende Klagbarkeitsdogmatik auf gesellschaftsrechtliche Treuepflichten, so setzt eine aus der Treuepflicht abgeleitete positive Stimmpflicht (1.) eine konkrete Beschlussvorlage (Bestimmbarkeit) und (2.) ein überwiegendes Interesse desjenigen voraus, der die Erfüllung der Treuepflicht in Natur einfordert. Dabei sind im Abwägungsprozess auch die Interessen des Schuldners der Treuepflicht zu berücksichtigen. Die Interessen des Gläubigers (bzw. der Gläubiger) werden typischerweise nur überwiegen, wenn diese eine Naturalerfüllung der Treuepflicht notwendig machen, wenn sie also im Verletzungsfall nicht vollständig in Geld ausgeglichen werden können.

Bei Lichte besehen sind diese Anforderungen weitgehend deckungsgleich mit den Voraussetzungen, welche die Rechtsprechung für eine positive Stimmpflicht aufstellt: Denn um zu ermitteln, ob die Stimmpflicht dringend geboten und dem betroffenen Gesellschafter zumutbar ist ${ }^{101}$, wird regelmäßig eine umfassende Interessenabwägung nötig sein.

Mustert man die eingangs erwähnten Beispiele durch ${ }^{102}$, wird man auf Basis der hier vertretenen These ebenso wie der BGH zu einer Bejahung der Klagbarkeit in den geschilderten Sanierungsfällen gelangen, gleichfalls in den Fällen der Ausschließungsklage, des Ausscheidens und der Änderung der Nachfolgeklausel.

Entgegen der Gruner + Jahr-Entscheidung des $\mathrm{BGH}^{103}$ wird man aber auch dem treuepflichtbasierten Wettbewerbsverbot des Geschäftsleiters einer Komplementär-Kapitalgesellschaft gegenüber der drittgeschützten Kommanditgesellschaft eine klagbare Unterlassungspflicht entnehmen können. Wie die Normierung des Eintrittsrechts in den $\S 88 \mathrm{AktG}, \S 112 \mathrm{HGB}$ typisierend zeigt, ist

99 Stürner, IZ 1976, 384, 389 ff.; ähnlich Köhler, AcP 190 (1990), 496, 506 f.

100 Köhler, AcP 190 (1990), 496, 508.

101 So BGH, Urt. v. 25.9.1986 - II ZR 262/85, BGHZ 98, 276, 280.

102 S. supra unter II.1.

103 S. supra unter II.2. 
eine Schadensersatzsanktion bei Wettbewerbsverstößen nämlich häufig ungenügend. Es kommt mithin gerade auf die Naturalerfüllung des Wettbewerbsverbotes an. Wenn die KG aber aufgrund der drittwirkenden organschaftlichen Treuepflicht einen eigenen Unterlassungsanspruch hat, kann ohne ihre Zustimmung kein wirksamer Dispens vom Wettbewerbsverbot erteilt werden. Mit anderen Worten: Es genügt nicht, dass die Komplementär-Kapitalgesellschaft als primäre Gläubigerin der organschaftlichen Treuebindung einen Dispens vom Wettbewerbsverbot erteilt; vielmehr ist auch die KG als drittgeschützte Gläubigerin des Wettbewerbsverbots an der Dispensentscheidung zu beteiligen ${ }^{104}$.

\section{Zusammenfassung in Thesen}

1. Im Hinblick auf die Klagbarkeit gesellschaftsrechtlicher Treuepflichten gibt es noch keine stringente Dogmatik. Unstreitig ist zwar, dass eine Treuepflichtverletzung auf Sekundärebene Schadensersatz auslösen kann (§280 Abs. 1 BGB). Ob die Treuepflicht aber auch schon auf der vorgelagerten Primärebene in Natur eingeklagt und durchgesetzt werden kann, wird von der herrschendem Meinung nur disparat von Fall zu Fall entschieden. Dies erhellen die Beispiele hinsichtlich der treuepflichtbasierten Stimmpflicht (etwa in Sanierungsfällen) und des treuepflichtbasierten Wettbewerbsverbots in Dreieckskonstellationen (etwa in einer Kapitalgesellschaft \& Co. KG zwischen dem Geschäftsleiter und der Kommanditgesellschaft).

2. Die gesellschaftsrechtlichen (= mitgliedschaftlichen/organschaftlichen) Treuepflichten sind ein Unterfall der Rücksichtspflichten und haben daher ebenso wie diese ihre normative Grundlage in den $\S \S 241$ Abs. 2, 242 BGB. Vor diesem Hintergrund scheint es konsequent, die Klagbarkeitsdogmatik, welche die Literatur für die schuldrechtlichen Rücksichtspflichten nach $\S 241$ Abs. 2 BGB entwickelt hat, auch auf die gesellschaftsrechtlichen Treuepflichten zu übertragen.

3. Rücksichtspflichten ( $\$ 241$ Abs. 2 BGB) sind nach herrschender Lehre auf Primärebene im Unterschied zu Leistungspflichten (§241 Abs. 1 BGB) nicht stets und vorbehaltlos, sondern nur unter zwei Voraussetzungen in Natur klagbar: (1.) Ihr Pflichtinhalt muss hinreichend bestimmbar sein. (2.) Ferner muss nach einer umfassenden Interessenabwägung ein überwiegendes schutzwürdiges Interesse desjenigen bestehen, der die Beachtung der Rücksichtspflicht in Natur einfordert. Ein überwiegendes Interesse ist regelmäßig anzunehmen, wenn es im Verletzungsfall nicht vollständig in Geld kompensiert werden kann, etwa weil der Schaden schwierig nachweisbar ist.

4. Überträgt man die für die schuldrechtlichen Rücksichtspflichten vertretene Klagbarkeitsdogmatik auf die gesellschaftsrechtlichen Treuepflichten, so ergeben sich im praktischen Ergebnis für die Fälle der positiven Stimm-

104 Ähnlich Cahn, Der Konzern 2007, 716, 725; M. Winter, Mitgliedschaftliche Treuebindungen im GmbH-Recht, 1988, S. 256; näher Weller, ZHR 175 (2011), 110, $140 \mathrm{f}$. 
pflicht keine nennenswerten Abweichungen gegenüber der bisher vertretenen Linie des BGH. Denn der BGH leitet aus der Treuepflicht nur dann eine positive Stimmpflicht $a b$, wenn diese dringend geboten und dem betroffenen Gesellschafter zumutbar ist. Anders verhält es sich jedoch für das Wettbewerbsverbot der Geschäftsleiter in der Kapitalgesellschaft \& Co. KG. Die Geschäftsleiter unterliegen bei Übertragung der allgemein für Rücksichtspflichten geltenden Klagbarkeitsdogmatik auf gesellschaftsrechtliche Treuepflichten nicht nur gegenüber der Komplementärgesellschaft, sondern über die drittwirkenden organschaftlichen Treuepflichten auch gegenüber der Kommanditgesellschaft einem Wettbewerbsverbot. Dieses kann von der Kommanditgesellschaft entgegen der Auffassung des BGH auch auf Primärebene in Natur in Form eines Unterlassungsanspruchs durchgesetzt werden. 